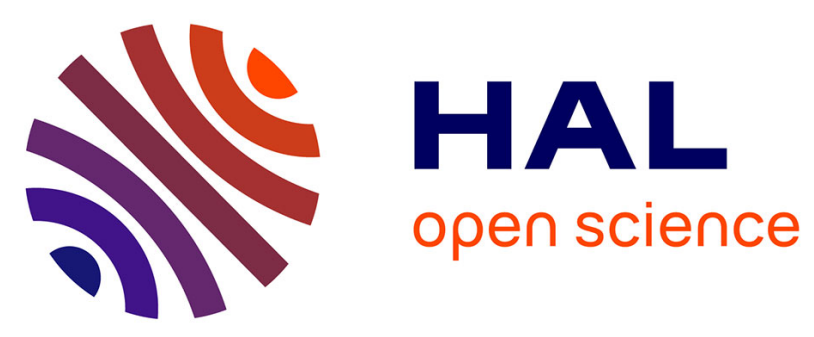

\title{
Les biotechnologies animales
}

\author{
L.M. Houdebine
}

\section{To cite this version:}

L.M. Houdebine. Les biotechnologies animales. Productions Animales, 1991, 4 (1), pp.81-88. hal00895927

\section{HAL Id: hal-00895927 \\ https://hal.science/hal-00895927}

Submitted on 1 Jan 1991

HAL is a multi-disciplinary open access archive for the deposit and dissemination of scientific research documents, whether they are published or not. The documents may come from teaching and research institutions in France or abroad, or from public or private research centers.
L'archive ouverte pluridisciplinaire HAL, est destinée au dépôt et à la diffusion de documents scientifiques de niveau recherche, publiés ou non, émanant des établissements d'enseignement et de recherche français ou étrangers, des laboratoires publics ou privés. 
INRA Prod. Anim. 1991, 4 (1), $81-88$

\section{L.M. HOUDEBINE}

INRA

Unité de Différenciation Cellulaire 78352 Jouy-en-Josas Cedex
Les

biotechnologies animales

\section{Les biotechnologies ouvrent des perspectives considérables pour l'élevage en permettant une maîtrise sans précédent du vivant. Des techniques dont la mise au point est en cours vont permettre de connaître beaucoup mieux les gènes des différentes espèces animales, de reproduire plus rapidement les animaux par clonage et de leur transférer des gènes nouveaux, d'accélérer et d'affiner les programmes de sélection.}

L'amélioration spectaculaire des productions animales à laquelle on assiste depuis les cinquantes dernières années est le résultat d'une double démarche: d'une part une meilleure utilisation des techniques traditionnelles d'élevage (alimentation, hygiène, reproduction...), d'autre part une application rapide de nouvelles techniques issues de la recherche (sélection génétique, contrôle de la reproduction, vaccination, alimentation...). Les très impor-

\section{Résumé}

Les progrès récents de la biologie et notamment ceux réalisés dans le domaine des acides nucléiques et des protéines permettent d'envisager de nouvelles stratégies très prometteuses pour l'élevage des animaux domestiques. Des sondes d'acide nucléique permettent la détermination du sexe chez l'embryon bovin précoce. Plus généralement la multitude de sondes qui vont être progressivement disponibles et la cartographie des génomes vont donner une bien meilleure connaissance des animaux. Il en résultera de nouvelles stratégies de sélection beaucoup plus fines et beaucoup plus rapides. La préparation en masse de peptides recombinants (antigènes d'agents pathogènes, hormones, cytokines, anticorps monoclonaux...), laisse envisager des interventions sur les animaux jusqu'alors réservées à des cas très particuliers. Les nouvelles techniques liées à la reproduction des mammifères (maturation des ovocytes in vitro, fécondation in vitro, développement des embryons précoces in vitro, duplication des embryons par clivage et par clonage) vont progressivement se superposer aux techniques déjà existantes (insémination artificielle, transfert d'embryons) et contribuer à raccourcir les cycles de reproduction et la durée des programmes de sélection génétique.

L’ingénierie génétique va permettre, dans les cas les plus favorables, de transmettre une protéine à un organisme entier sous la forme d'un gène actif. Des gènes peuvent être transférés dans les cellules somatiques des organismes entiers (thérapie génique) ou dans les embryons précoces (transgénèse). La thérapie génique peut permettre de ne plus devoir administrer un polypeptide de manière répétée à un animal et la transgénèse conduit à l'obtention de nouvelles lignées d'animaux présentant des caractères génétiques nouveaux définis par les transgènes. Ces interventions, qui sont les plus radicales et les plus prometteuses, ne pourront devenir une réalité significative dans les élevages qu'à la fin de ce siècle. tants progrès que la recherche a accomplis dans la plupart des domaines de la biologie depuis vingt ans, et plus particulièrement depuis dix ans dans la génétique moléculaire, ont conduit à repenser profondément les programmes de recherches appliqués aux êtres vivants. Il ne fait en effet plus de doute que l'exploitation des organismes vivants, microorganismes, végétaux et animaux, va, d'une manière ou d'une autre, être bouleversée par l'apport de ces nouvelles découvertes et plus particulièrement par l'ingénierie génétique qui a fait son entrée dans de multiples secteurs de la biologie (étude de l'expression génétique, biologie cellulaire, ingénierie des protéines, physiologie...). Il est d'ores et déjà possible de classer ces nouvelles approches en plusieurs catégories: $1^{\circ}$ ) l'observation fine des caractères génétiques des animaux à l'aide de sondes d'acide nucléique, suivie d'une sélection déterminée par ces caractères, $2^{\circ}$ ) l'intervention sur les animaux par l'administration de protéines recombinantes (antigènes d'agents pathogènes, hormones, facteurs de croissance, facteurs de différenciation, anticorps monoclonaux...), $3^{\circ}$ ) la diffusion accélérée du génome entier des animaux par la maîtrise de techniques de la reproduction $4^{\circ}$ ) le transfert d'un caractère génétique nouveau à un organisme entier par l'intermédiaire de la thérapie génique et de la transgénèse.

La révolution dans l'élevage des animaux que laissaient prévoir les découvertes de la biologie est désormais en marche. La complexité même des organismes animaux rend toutefois cette démarche relativement lente et difficile sans pour autant inciter au pessimisme. Il est possible de faire le point sur les réalisations en cours et de dresser un inventaire des perspectives qui s'offrent d'ici la fin de ce siècle. 


\section{L'observation des animaux à l'aide des sondes moléculaires}

La sélection génétique repose essentiellement sur le repérage d'un caractère nouveau chez les individus d'un troupeau, suivi de la transmission de ce caractère à la descendance par les techniques classiques de reproduction. Toute l'efficacité de cette méthode tient dans la perception que l'on peut avoir des caractères génétiques étudiés. Une fonction biologique est contrôlée dans la grande majorité des cas par un relativement grand nombre de gènes qui ne s'expriment pas forcément dans la même cellule ou le même tissu. Les communications intercellulaires assurées entre autres par les hormones assurent la cohérence de l'ensemble. Un caractère génétique donné a donc lui-même peu de chance de ne dépendre que d'un seul gène au sens moléculaire du terme.

L'examen des protéines, en particulier à l'aide d'électrophorèse, permet d'établir dans certains cas favorables des corrélations entre un caractère génétique donné et la présence d'une bande dans un électrophorégramme.

Des sondes d'acide nucléique qui peuvent, à la base près, révéler aisément une mutation dans un gène constituent des outils incomparablement plus précis pour procéder à une sélection génétique fine.

Il convient de ce point de vue de faire des distinctions entre les différents types de sondes que l'on peut utiliser. Certaines correspondent directement à la séquence d'un gène codant pour une protéine bien définie tandis que d'autres détectent des régions non fonctionnelles ou supposées telles du génome. Les informations apportées par ces deux types de sondes ne seront évidemment pas tout à fait de même nature. Dans les cas les plus favorables, les sondes RFLP (restriction fragment length polymorphism) peuvent être utilisées. Cette méthode repose sur la présence d'un site de restriction (site où une nucléase coupe spécifiquement l'ADN] différant entre deux variants de même gène. Plus généralement, les sondes ASO (allele specific oligonucleotides) permettent de faire une distinction entre deux séquences du même gène ne différant que par un seul nucléotide (Caskey 1987). Il existe dans le génome de la plupart des eucaryotes des séquences qui sont répétées un grand nombre de fois (ADN satellite) et qui sont réparties apparemment au hasard dans l'ADN cellulaire. Ces séquences, qui n'ont semble-t-il pas de fonction précise, en tout cas en ce qui concerne l'expression génétique, ne sont pas soumises à une forte pression de sélection. Elles varient donc d'un individu à l'autre. Elles peuvent être repérées à l'aiede de sondes qui sont quasi-universelles (Jeffrey et al 1985) et donc permettre ainsi de suivre très finement et avec une remarquable précision la filiation entre individus.

Les méthodes d'hybridation moléculaire dans lesquelles sont utilisées ces sondes sont d'une très grande sensibilité et quelques microgrammes d'ADN d'un individu suffisent pour le caractériser. Des quantités encore beaucoup plus faibles peuvent même être utilisées lors- que la technique d'amplification spécifique de gènes PCR (Polymerase chain reaction) est utilisée. Théoriquement une seule cellule peut alors suffire à détecter un gène donné présent à l'état d'une seule copie par génome haploïde. Il est donc théoriquement possible avec les sondes nucléiques de déterminer un caractère génétique donné, ou tout au moins une séquence spécifique d'ADN, à n'importe quel moment de la vie d'un animal, à partir de la semence, d'un embryon, du sang ou d'un organe. Cette nouvelle manière d'observer les animaux permet potentiellement d'atteindre une précision sans précédent et de gagner un temps considérable dans les processus de sélection puisque le produit d'un gène ou son effet phénotype n'a plus besoin d'être mesuré dès lors qu'une corrélation a pu être établie entre la séquence d'un fragment d'ADN donné repérable par une sonde spécifique et un caractère génétique donné (Bechmann et Soller 1987). Les caractéristiques d'un géniteur pourront donc dans le meilleur des cas être connues dès sa naissance. La cartographie systématique du génome de quelques animaux domestiques comme le porc et le bovin qui a été entreprise va donner une dimension encore supérieure à la connaissance que nous avons de ces animaux (voir l'article de J. Gellin et F. Grosclaude dans ce numéro). La multiplication des sondes qui vont être mises progressivement à la disposition des généticiens va rapidement rendre extrêmement complexe la gestion de ces informations. Une gestion informatisée d'un type nouveau va donc devoir être mise en place.

Quelques sondes sont d'ores et déjà disponibles et commencent à faire l'objet d'applications.

\section{Les sondes ADN spécifiques du chromosome $\mathrm{Y}$ bovin}

Il est évidemment souhaitable de connaître le plus tôt possible le sexe d'un embryon de mammifère pour pouvoir gérer au mieux un troupeau. Des recherches ont été entreprises il y a plusieurs années pour tenter d'identifier des antigènes de surface spécifiques des cellules des embryons mâles de mammifères. Ces recherches se sont avérées délicates et la notion même d'antigène spécifique de l'embryon mâle chez les mammifères est incertaine. Le chromosome Y, spécifique du mâle, doit évidemment contenir des séquences qui lui sont spécifiques. Une recherche systématique a permis à plusieurs groupes d'identifier de telles séquences qui ne sont pas toutes identiques. Des sondes spécifiques de l'embryon mâle bovin et porcin sont maintenant disponibles (Léonard et al 1987, Mc Gran et al 1988). Une telle sonde doit pouvoir être utilisée d'une manière simple n'impliquant pas l'usage de marqueurs radioactifs. Elle doit également pouvoir donner la réponse en quelques heures seulement à partir de quelques cellules prélevées sur l'embryon, de manière à permettre son transfert ou sa congélation sans perte de viabilité. Ces problèmes sont résolus grâce à l'utilisation de la technique de PCR évoquée précédemment et un diagnostic du sexe est donc possible pour les bovins (figure 1). Une application à des ani- 
Figure 1. Utilisation de la sonde d'acide nucléique spécifique du chromosome $Y$ de bovin pour le sexage des embryons. Une séquence spécifique de l'ADN provenant de quelques cellules de l'embryon est amplifiée spécifiquement par la technique de PCR et visualisée par électrophorèse.

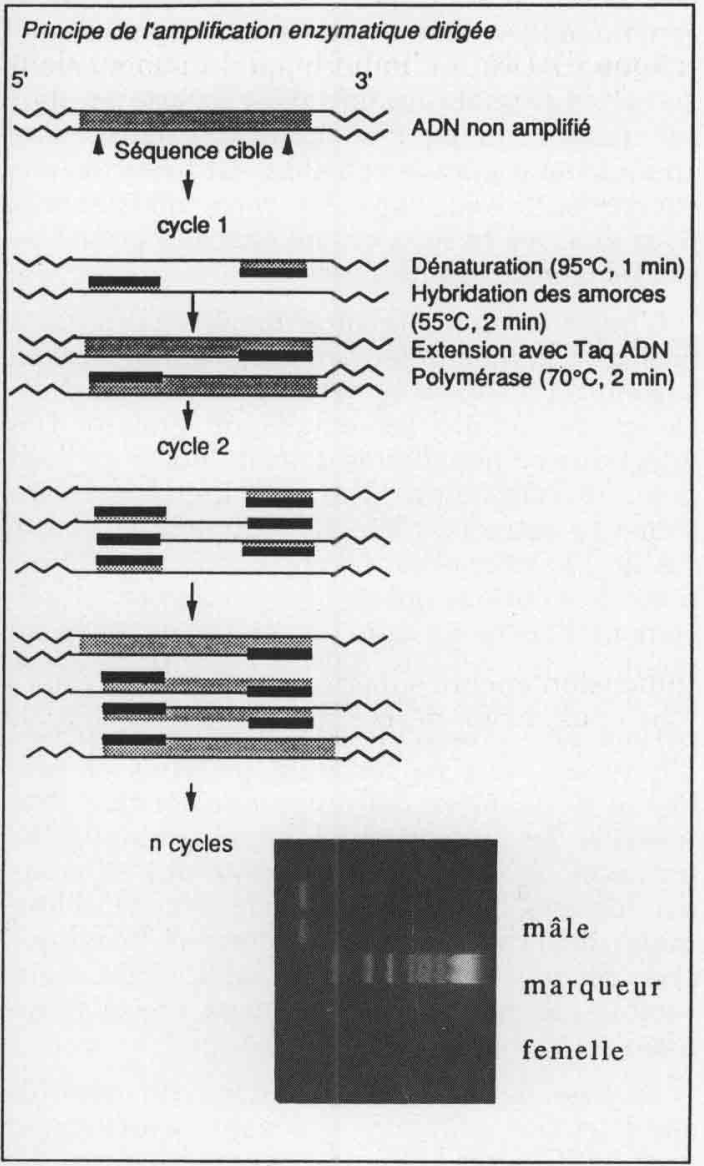

maux de plus petite taille peut se heurter au prix de revient du test.

\section{Les gènes des protéines du lait}

Le lait représente $20-30 \%$ des protéines consommées dans les pays développés. Les protéines du lait des ruminants existent sous la forme de plusieurs variants dont les propriétés sont différentes et confèrent au lait des qualités variables, en particulier pour préparer les fromages. L'inventaire de ces variants a été fait pour un certain nombre de races et une sélection génétique est possible (Grosclaude 1988). La stratégie classique de sélection impose que le caractère génétique de la protéine examinée soit déterminé par examen du lait. Un tel examen ne peut évidemment se faire que chez des femelles en lactation. La technique d'évaluation des variants repose par ailleurs sur des électrophorèses qui peuvent ne pas révéler certaines variations dans la composition des protéines et dans la structure des gènes correspondants. Des sondes d'acide nucléique sont maintenant disponibles et elles permettent de révéler la présence de tel ou tel variant aussi bien chez le mâle que chez la femelle dès les stades précoces de leur vie. Ainsi peuvent être potentiellement sélectionnés les animaux portant les variants $\mathrm{KB}$ et $\beta \mathrm{B}$ des caséines qui confèrent au lait une meilleure aptitude fromagère et le variant $\beta$-lactoglobuline $B$ qui est moins abondant que le variant $A$ et qui s'accompagne d'une plus haute teneur du lait en caséine.

\section{Les gènes de résistance aux maladies}

Il est bien connu qu'une certaine proportion d'individus d'un troupeau présente spontanément une résistance à certaines maladies. Cette protection naturelle est en général héréditaire. Une corrélation entre la présence de certains gènes et le caractère de résistance a pu être établie dans quelques cas. Une telle corrélation ne signifie pas nécessairement que le gène repéré a un lien fonctionnel direct avec le caractère de résistance. Il permet néanmoins de procéder à une sélection. C'est le cas des poulets résistants spontanément à la maladie de Marek. Ces poulets ont l'haplotype B21 et ils sont résistants lorsqu'ils sont homozygotes B21/B21. Une sélection sur le caractère B21 est donc possible grâce à une sonde nucléique spécifique (Cauchy et al 1987). L'identification des gènes responsables de cette résistance est en cours. Elle pourrait permettre de pouvoir conférer la résistance à des lignées d'oiseaux par la transgénèse et non plus seulement par la voie classique de la transmission à la descendance.

\section{Les séquences virales des génomes d'oiseaux}

Un certain nombre d'animaux portent dans leur génome des séquences virales et en particulier rétrovirales qui sont des reliquats d'infections et qui ont perdu leurs caractères pathogènes ou fonctionnels à la suite de mutations spontanées. Des corrélations ont pu être établies entre la présence de certaines de ces séquences et le taux de croissance des animaux. Certaines séquences virales, qu'elles soient exprimées ou non, doivent donc, pour des raisons inconnues, légèrement perturber l'expression génétique des cellules qui les portent. La détection de ces séquences est possible à l'aide de sondes nucléiques spécifiques et une élimination progressive des animaux porteurs peut être envisagée.

\section{Le diagnostic de gestation}

La bonne gestion d'un troupeau est facilitée si l'on peut savoir rapidement si une femelle est gestante ou non. Des diagnostics fiables existent déjà. Ils reposent sur la mesure des hormones de la gestation et en particulier de la progestérone. Ces tests ne sont pas tous utilisables dans les premiers stades de la gestation. Une détermination des signaux délivrés précocément par l'embryon est susceptible de donner une réponse plus satisfaisante à cette question. Une famille de protéines présente exclusivement dans le sang des vaches gestantes a été récemment mise en évidence. Un test radioimmunologique permet un diagnostic de gestation (Camous et al 1989).

D'autres sondes basées sur l'utilisation d'anticorps polyclonaux ou monoclonaux sont utilisables pour de multiples usages en particulier pour déceler la présence d'agents pathogènes. Ces sondes constituent un complément intéres-
Enzyme de restriction : Nucléase qui coupe l'ADN en un site spécifique défini par la séquence des bases: les fragments d'ADN obtenus par ces enzymes sont appelés fragments de restriction.

RFLP (restriction fragment length polymorphism) : Propriété qu'a l'ADN de différents allèles de générer des fragments de restriction de taille variable. Ce polymorphisme reflète directement des variations de la séquence primaire de l'ADN.

PCR (polymerase chain reaction): Amplification spécifique in vitro d'une séquence d'acide nucléique à l'aide d'une polymérase et de deux amorces oligonucléotidiques qui en cadrent la région à amplifier. 
sant aux sondes nucléiques étant donnée leur excellente spécificité et leur relative facilité d'utilisation.

\section{L'utilisation des protéines recombinantes à usage vétérinaire}

L'utilisation de molécules ayant une activité biologique comme les hormones excluait presque totalement les protéines jusqu'au développement du génie génétique. Les hormones protéiques sont en effet relativement difficiles à préparer massivement à partir des organes animaux en raison de leur rareté. Les hormones gonadotropes sont toutefois déjà largement utilisées. La synthèse chimique des peptides est possible mais son prix de revient devient rapidement très prohibitif au delà de 40 acides aminés. Il est cependant possible de fabriquer industriellement des facteurs de libération des hormones hypophysaires comme le TRH, le LHRH ou le GRF. Les techniques de clonage des gènes permettent de plus d'identifier beaucoup plus facilement des facteurs de régulation de l'activité cellulaire et de disposer des séquences d'ADN correspondantes. Des protéines recombinantes (insuline, hormone de croissance, etc...) sont fabriquées industriellement pour des usages cliniques. Le nombre de ces protéines est en très rapide développement. Ces protéines peuvent être obtenues à partir de bactéries de levures, de champignons, de plantes, de cellules d'insectes, du sang et du lait de mammifères transgéniques. Une applica-

Figure 2. Schéma récapitulatif des différentes techniques appliquées aux embryons des mammifères domestiques.

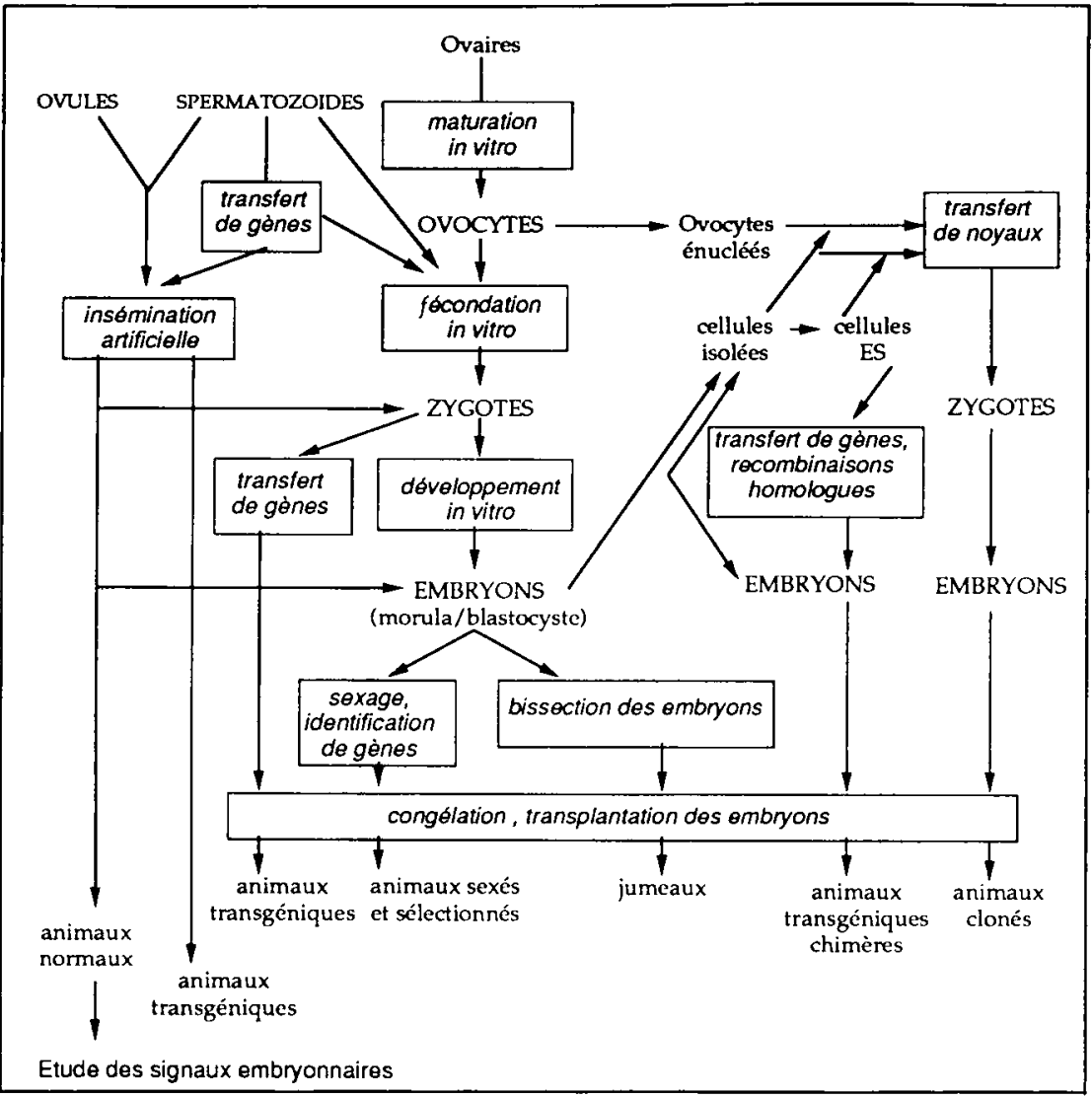

tion aux animaux est devenue possible. Il est admis depuis 50 ans que des injections d'hormone de croissance augmentent très significativement la production laitière des ruminants domestiques (15-25\%). Cette observation n'a pu avoir de développement significatif tant que la protéine n'avait pu être obtenue par génie génétique. Il est admis que ce gain de productivité, qui devrait se traduire par un abaissement du prix de revient du lait, n'est néfaste ni pour les animaux ni pour les consommateurs. Une application à grande échelle n'est toutefois pas encore autorisée dans les pays occidentaux pour diverses raisons qui ne sont pas pour l'essentiel d'ordre scientifique.

L'hormone de croissance injectée à des porcs favorise légèrement leur croissance. Elle permet surtout de favoriser le développement du muscle et de réduire les dépôts de graisse. Des injections systématiques de cette hormone sont donc envisageables. Elles sont toutefois relativement contraignantes étant donnée la courte vie de l'hormone dans l'organisme. Des injections de solutions qui ne libèrent que progressivement l'hormone sont en cours de mise au point. La transgénèse qui est capable de générer des animaux exprimant le gène de la $\mathrm{GH}$ permet de s'affranchir des injections répétées d'hormone. Elle ne résoudra toutefois ce problème de manière définitive que lorsque l'expression des transgènes GH sera bien maîtrisée, un excès de GH altérant la santé des animaux (Houdebine 1990). Une surabondance d'hormone de croissance est également bénéfique pour des poissons comme les salmonidés, mais, semble-t-il, pas pour les oiseaux et les ruminants.

La liste des protéines exerçant un contrôle sur l'activité cellulaire qui sont actuellement préparées pour des usages vétérinaires est encore réduite. On peut citer les interférons $\alpha$ et $\beta$, l'interleukine-2 et la FSH. Cette liste ne peut que s'allonger. Un des avantages d'une telle approche thérapeutique est évidemment que les protéines sont instables dans le sang et de toute manière inactivées lors de la cuisson et la digestion de la viande. Elles sont donc, contrairement à certains stéroïdes par exemple, d'une totale innocuité pour le consommateur.

\section{Les vaccins de la nouvelle génération}

Ce chapitre est traité dans l'article de J.M. Aynaud, page 89.

\section{Le contrôle de la reproduction}

Un certain nombre de techniques contribuent déjà en routine à améliorer la reproduction des animaux domestiques et à accélérer la sélection génétique (insémination artificielle, synchronisation des chaleurs et des mise-bas, transfert, congélation et clivage des embryons). De nouvelles techniques permettant d'obtenir des embryons précoces en très grand nombre sont en passe d'être maîtrisées chez plusieurs espèces domestiques, en particulier chez les ruminants. Ces embryons pourront être utilisés pour des études à caractère fondamental, pour 
la sélection génétique, pour le clonage des embryons par transfert nucléaire et pour la transgénèse (figure 2).

\section{La fécondation in vitro}

Les embryons produits in vivo après superovulation ef fécondation peuvent être obtenus en relative abondance. Ce matériel biologique est toutefois hétérogène dans la mesure où les moments précis de l'ovulation et de la fécondation ne peuvent être connus avec précision in vivo. Les embryons produits de cette manière sont de plus d'un prix relativement élevé. Il est possible d'obtenir in vitro une maturation des ovocytes à partir d'ovaires collectés sur des vaches à des stades physiologiques quelconques au moment de l'abattage. Il est de même possible de réaliser in vitro la capacitation du sperme, la fécondation et le développement précoce jusqu'au stade de morula (8-32 cellules) chez les ruminants domestiques (Crozet 1990). Le rendement de cet ensemble d'opérations s'il est encore relativement faible est en constante amélioration et tout laisse penser qu'il est en passe d'avoir un véritable impact sur l'élevage.

\section{Le clonage des embryons}

Le transfert du noyau d'une cellule d'un embryon précoce dans le cytoplasme d'un embryon au stade une cellule ou d'un ovocyte peut conduire au développement de cet embryon reconstitué et à la génération d'un organisme entier. Cette opération qui consiste à cloner un animal (et donc à le multiplier à l'infini sans passer par le cycle normal de la reproduction) a été réalisée depuis longtemps chez les batraciens et beaucoup plus récemment chez les mammifères. Il est généralement admis que c'est la différenciation du noyau beaucoup plus rapide chez les vertébrés supérieurs qui rend le clonage plus difficile chez ces espèces. Le mécanisme de la reprogrammation du noyau qui passe d'une cellule d'un embryon dans le cytopolasme d'un ovocyte est, pour l'essentiel, inconnu. Le clonage chez les mammifères comprend les étapes suivantes: énucléation de l'ovocyte par microchirurgie, introduction d'une cellule embryonnaire dans l'embryon au voisinage de l'ovocyte par micromanipulation, fusion des membranes de l'ovocyte et de la cellule embryonnaire par un choc électrique, activation du nouvel embryon (qui mime l'activation normalement provoquée par le spermatozoïde et qui induit le développement de l'embryon). Cette technique a été réalisée avec succès chez les bovins, les porcins, les ovins et le lapin (Marx 1988; Prather et al 1989). Elle deviendra une réalité dans les élevages lorsque son rendement global aura été amélioré.

La congélation des ovocytes receveurs de noyaux et des noyaux de cellules donneuses avant l'opération de transfert apporterait par ailleurs une grande souplesse à l'ensemble des opérations si elle arrivait à être maîtrisée. Les caractéristiques génétiques d'un individu ne sont en effet que très partiellement connues avant sa naissance. Une mise en réserve par congélation des blastocystes donneurs de noyaux, jusqu'à l'évaluation des propriétés du patrimoine génétique de ces noyaux chez un animal adulte, permettrait de ne réaliser le clonage que pour les individus les plus intéressants.

\section{La transgénèse chez les animaux}

Il y a bientôt 10 ans, la preuve a été donnée qu'il était possible d'introduire un gène isolé dans un embryon et de faire en sorte que ce gène étranger soit exprimé chez l'animal adulte et transmis à sa descendance. Cette opération, appelée transgénèse, a maintenant été réalisée chez toutes les catégories d'animaux : insectes, vers, mollusques, poissons, poulets, petits et gros mammifères.

\section{Les méthodes de transgénèse (figure 3)}

La microinjection directe du gène en solution dans l'un des pronucléi de l'embryon de mammifère au premier stade de son développement a été la première méthode utilisée avec succès. Elle reste la seule pratiquée à grande échelle. Cette méthode est néanmoins pénible puisqu'elle implique que les embryons aient été isolés, microinjectés puis réintroduits dans une mère adoptive. Elle reste relativement peu efficace puisqu'environ $2 \%$ seulement des embryons de souris manipulés deviennent des adultes transgéniques. Le rendement devient nettement plus faible chez les autres espèces de mammifères et il est admis que pour obtenir un animal transgénique, il faut en moyenne 10 souris (donneuses et receveuses d'embryons) 20 truies, 20 brebis et 40 vaches. Ces rendements sont loin d'être atteints en routine dans tous les laboratoires. Il ne faut pas oublier par ailleurs qu'une partie non négligeable des animaux transgéniques n'a qu'un intérêt très réduit dans la mesure où ils n'expriment pas ou très peu le transgène qui s'insère dans des régions totalement imprévisibles du génome des animaux hôtes (Pursel et al 1989; Brem 1990).

Chez les invertébrés et les vertébrés inférieurs et notamment chez les poissons, des injections d'ADN en grande quantité dans le cytoplasme des embryons au stade une cellule permet d'obtenir des animaux transgéniques avec un très haut rendement. Ces animaux sont cependant mosaïques dans presque tous les cas (Chourrout et al 1990).

L'utilisation de rétrovirus jouant le rôle de vecteur a été proposée comme alternative indispensable chez des espèces comme les oiseaux chez lesquels la manipulation des embryons précoces n'est pas assez maîtrisée pour que des microinjections directes de gène puissent être envisagées. (Bosselman et al 1989). Une technique permettant le développement des embryons de poulet in vitro a toutefois ouvert des perspectives prometteuses (Sang et Perry 1989).

L'utilisation de vecteurs non viraux mais capables de se repliquer de manière autonome à une haute fréquence sans être intégrés au génome de l'animal hôte ouvrirait des perspectives intéressantes dans la mesure où elle améliorerait de manière considérable le rendement
La transgénèse offre beaucoup de possibilités mais elle se heurte à des problèmes techniques et à la relative méconnaissance des gènes à transférer.

Protéine recombinante : Protéine synthétisée par un organisme vivant sous la direction d'un gène qui lui a été transféré.

Mosaïque : Un animal est mosaique pour un transgène si une partie seulement de ses cellules $y$ compris de ses cellules germinales) abrite le transgène. De même, un animal chimère qui a reçu des cellules d'un autre animal au stade embryonnaire est un animal mosaïque puisque ses cellules n'ont pas toutes le même génome. 
Figure 3. Les différentes méthodes utilisables pour obtenir des animaux transgéniques.

- La microinjection. L'embryon est maintenu par une pipette dans le champ d'un microscope. La microinjection consiste à introduire, à l'aide d'une aiguille en verre, le gène étranger dans un des pronoyaux.

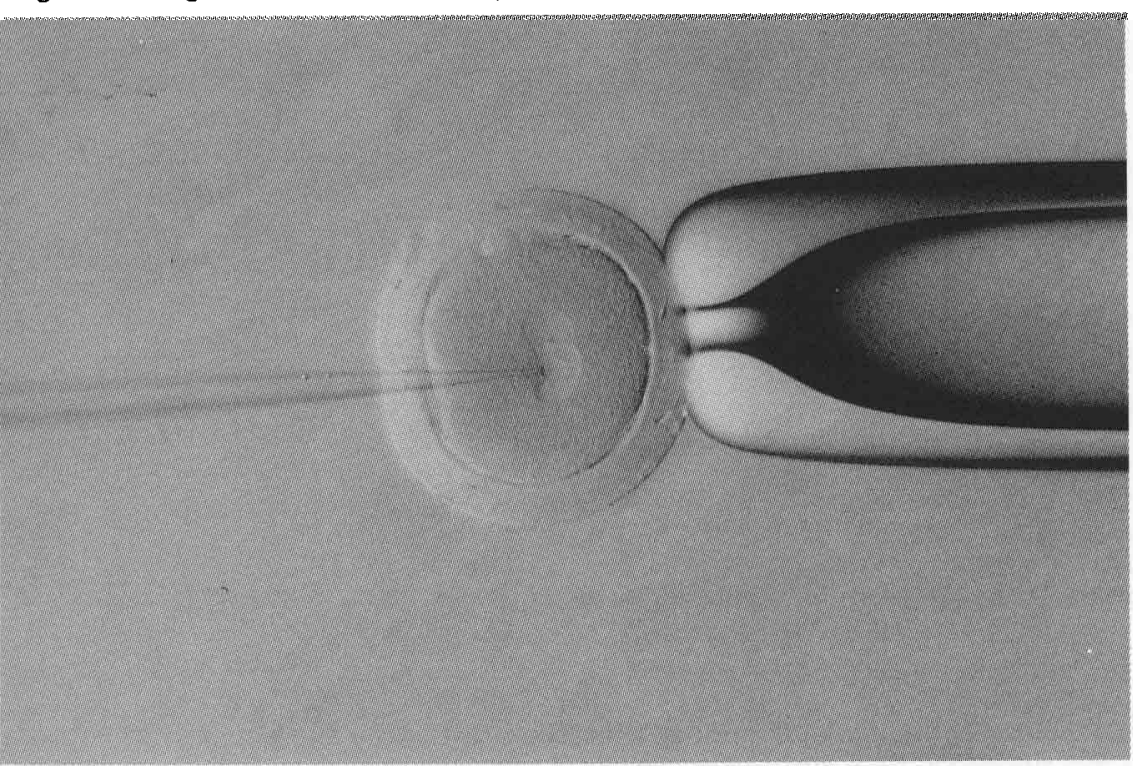

- Utilisation des vecteurs rétroviraux.



- Utilisation des cellules souches embryonnaires (ES).

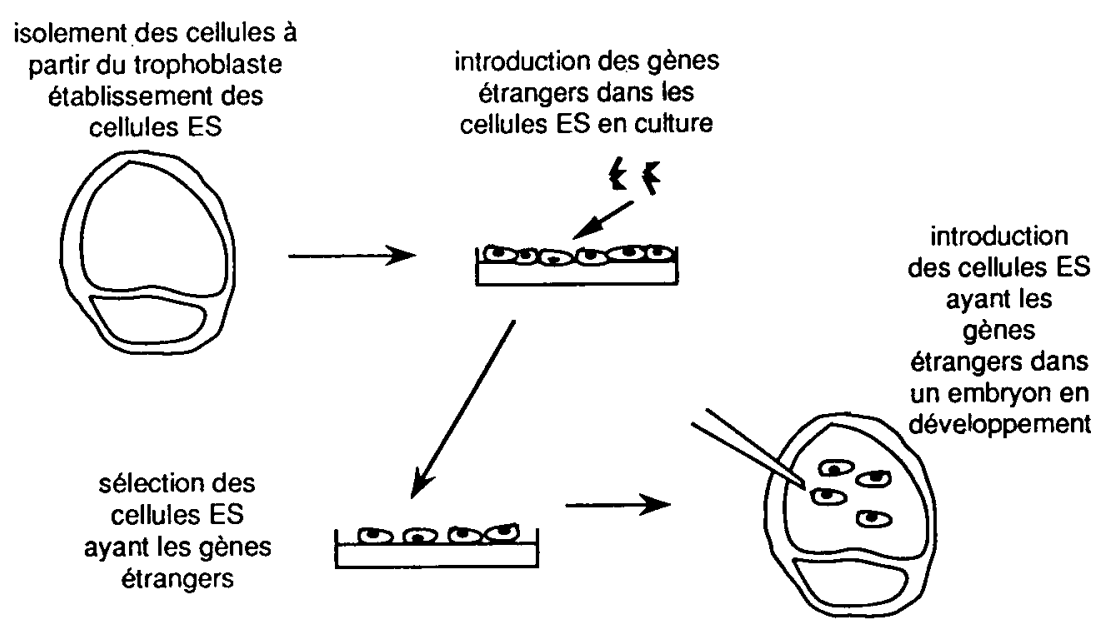

de la transgénèse. Les vecteurs de ce type actuellement disponibles ne sont pas d'une efficacité suffisante pour être réellement utilisables.

Une autre méthode qui a été couronnée de succès chez la souris consiste à utiliser des cellules souches embryonnaires (cellules ES) qui peuvent être cultivées dans des conditions où elles ne se différencient pas et où elles gardent donc leur totipotence. Ces cellules peuvent in vitro recevoir un gène étranger, être sélectionnées puis réintroduites au milieu des cellules d'un embryon en cours de développement. Cette opération conduit à la génération d'animaux chimères et donc mosaïques pour le transgène dans la mesure où au mieux une partie seulement des cellules de l'adulte (y compris de ces cellules germinales) a le gène étranger. Les animaux transgéniques de la génération suivante sont homogènes de ce point de vue et toutes leurs cellules ont le transgène (Robertson et al 1986). Tout laisse à penser que cette technique est transposable aux gros mammifères. L'utilisation des cellules ES paraît de plus actuellement la seule qui permette de substituer très précisément un gène endogène par un gène homologue muté plutôt que de surajouter un gène étranger au génome de l'hôte (Mansour et al 1988). Chez les oiseaux, des chimères réalisées à partir de cellules d'oeufs fraîchement pondus permettent d'envisager des choses semblables (Petitte et al 1990).

L'introduction directe d'un gène dans les spermatozoïdes avant la fécondation a été proposée mais ne peut être reproduite telle qu'elle a été décrite (Brinster et al 1989). Des modifications basées sur l'utilisation d'un tampon particulier et de l'électroporation semblent donner des résultats très prometteurs respectivement chez le poulet (E. Revel, résultats non publiés) et chez les bovins (C.M.A. Sirard, résultats non publiés)

\section{Les applications possibles de la transgénèse}

La transgénèse est devenue un outil de tout premier ordre pour des études fondamentales sur les mécanismes de contrôle de l'expression des gènes et sur leurs rôles dans le développement et le fonctionnement des organes. C'est essentiellement la souris qui est utilisée pour ces études. La souris et plus rarement d'autres animaux transgéniques commencent également à être considérés comme d'excellents modèles vivants pour des études cliniques et pharmacologiques.

Il est maintenant prouvé que des animaux transgéniques peuvent être utilisés comme fermenteurs vivants pour produire des protéines rares d'intérêt clinique ou vétérinaire dans leur lait (Clark et al 1989) ou leur sang (Massoud et al 1990). Si ce procédé devait atteindre le stade industriel il donnerait sans aucun doute une grande valeur aux animaux concernés. Un nombre relativement très faible suffirait toutefois à produire les protéines recombinantes et il est vraisemblable que ce sont les industries pharmaceutiques plutôt que les éleveurs qui tireraient un véritable profit de cette opération. 
Le véritable impact de la transgénèse sur l'élevage aura lieu lorsque les caractéristiques phénotypiques des animaux auront pu être modifiées par cette technique. Le transfert de gènes conférant aux animaux une résistance contre les maladies (vaccination génétique) paraît actuellement de ce point de vue l'opération la plus abordable. L'expression de ces gènes de résistance ne modifie pas de manière notable la physiologie des animaux dans la majorité des cas. Il n'en est pas de même des gènes codant pour les hormones de croissance par exemple. L'expression incontrôlée des transgènes des hormones de croissance perturbe en effet grandement la vie des animaux transgéniques chez la plupart des espèces (Houdebine 1990).

Un transgène contrôlé à volonté pour ne s'exprimer qu'à certains moments de la vie de l'animal est de toute évidence nécessaire. Il n'existe aucune impossibilité théorique à cet égard mais force est de constater que ce problème n'est pas encore résolu.

Parmi les projets qui sont à l'étude et qui ont des chances raisonnables de conduire à l'obtention de nouvelles lignées d'animaux on peut mentionner les expériences qui visent à augmenter la production de laine chez les moutons. La croissance de la laine est en effet limitée par la disponibilité de la cystéine chez l'animal. Le transfert des gènes de microorganismes permettant la transformation de la sérine en cystéine rendrait les moutons moins dépendant de la cystéine de la ration (Rogers 1990).

Des études conduites chez les animaux monogastriques permettent d'envisager le transfert des gènes bactériens qui permettraient la synthèse des acides aminés essentiels comme la lysine (Rees et al 1990).

La composition du lait (qui représente 20 à $30 \%$ des protéines consommées dans les pays riches) peut être changée par la transgénèse. La transgénèse peut théoriquement permettre

$\left.1^{\circ}\right)$ de modifier la proportion des différentes caséines et ainsi d'augmenter la qualité fromagère des laits,

$2^{\prime \prime}$ d'abaisser le taux de lactose et de $\beta$-lactoglobine qui provoquent des réactions allergiques,

$3^{\circ}$ ) de materniser les laits en y exprimant le gène de la transférine,

$\left.4^{\circ}\right)$ d'abaisser le taux de lipides ou de modifier leurs compositions en réduisant l'expression du gène de l'acétyl CoA carboxylase et en transférant le gène de la $\Delta$-2-désaturase,

$\left.5^{\circ}\right)$ d'augmenter l'état sanitaire de la glande mammaire en y faisant s'exprimer des protéines qui ont la propriété d'inactiver des agents bactériens (lysozyme, lysostaphine, anticorps monoclonaux, etc...).

Plus généralement, il paraît concevable de transférer par la transgénèse à des animaux autres que des moutons des gènes comme le gène Boroola de prolificité. Il est bien clair toutefois que ceci ne sera possible que lorsque la séquence du ou des gènes qui confèrent le caractère Boroola aura été identifiée et isolée. Il n'est pas certain pour autant que le transfert de ce ou ces gènes se traduira automatiquement par une prolificité accrue des animaux transgé- niques. En effet, trop peu de choses sont encore connues en ce qui concerne le mécanisme d'action du gène Boroola en terme cellulaire et moléculaire et les effets les plus inattendus sont possibles avec un tel transgène.

Le transfert de gènes aux animaux domestiques ne doit pas être restreint à la transgénèse. Il est en effet concevable de transférer des gènes aux cellules somatiques d'un animal adulte qui bénéficie alors des protéines pour lesquelles il code. Une autre approche applicable aux ruminants peut consister à modifier les bactéries du rumen par génie génétique. Cette opération potentiellement très efficace est délicate à plusieurs titres. Rien ne prouve en effet que les bactéries recombinées s'implanteront durablement dans le rumen. Il sera par ailleurs difficile de contrôler la diffusion dans l'environnement de ces bactéries.

\section{Conclusion}

Cet inventaire des recherches en cours n'est certainement pas complet. Il n'en est pour autant probablement pas moins excessif. Il est en effet probable que certains des projets actuels ne conduiront à aucune application parce que trop difficilement réalisables ou rendus inutiles en face d'autres techniques plus simples. La sélection génétique, en particulier chez les espèces animales dont le cycle de reproduction est court, peut avoir conduit aux changements attendus avant que la transgénèse ou les vaccins de la nouvelle génération ne soient devenus une réalité. Il n'en reste pas moins vrai qu'un gène n'est transférable d'une espèce animale à une autre autrement que par la transgénèse.

Le peu de succès rencontré par la transgénèse animale en terme d'application peut laisser planer des doutes sur l'importance potentielle réelle de cette approche. Les succès remportés chez les animaux de laboratoire pour des études fondamentales et chez les plantes pour certaines applications invitent plutôt à l'optimisme. Relativement peu de tentatives n'ont encore été faites chez les animaux domestiques et les investissements dans ce domaine restent donc justifiés. La prochaine décennie verra donc très probablement des changements dans la plupart des domaines des biotechnologies animales. Ces changements ne seront que relativement progressifs et les retombées des études actuelles n'atteindront probablement leur véritable dimension qu'au début du siècle prochain (Massey 1990).

\section{Références bibliographiques}

BECKMANN J.S., SOLLER M., 1987. Molecular markers in genetic improvement of farm animals. Biotechnology, $5,573-576$.

BOSSELMAN R. HSU R.Y. BOGGS T, HU S., BUSZEWSKI J., OU S., LOZAR L., MARTIN F., GREEN C.. JACOBSEN F., NICOLSON M., SCHULTZ J.A., SEMON K.M., RISHELL W, STEWART R.G. 1989. Germe line transmission of exogenous genes in the chicken. Science, 243, 533-535
Vaccination génétique : Opération qui consiste à transférer un gène de résistance à une maladie qui agit depuis l'intérieur de la cellule sans faire nécessairement intervenir le système immunologique de défense.

Electroporation : Opération qui consiste à créer des pores dans la membrane des cellules à l'aide d'impulsions électriques qui permettent à l'ADN en solution de pénétrer dans les cellules. 
BREM G., 1990. State of the art, limitations and prospective of gene transfer in domestic mammals. 6" Réunion de l'Association Européenne de Transfert d'Embryons, 97103.

BRINSTER R.L., SANDGREN E.P., BEHRINGER R.R. PALMITER R.D. 1989. No simple solution for making transgenic mice. Cell. 59, 239-241.

CAMOUS S., CHARPIGNY G., GUILLOMO'T' M., MARTAL J., SASSER R.G., 1989. Purification d'une protéine bovine spécifique de la gestation chez la vache par chromatographie liquide à haute performance. XXVIème Réunion de la Société Française pour l'Etude de la Fertilité (sous presse).

CASKEY C.T., 1987. Disease diagnosis by recombinant DNA methods. Science, 236, 1223-1228.

CHOURROUT D., GUYOMARD R., HOUDEBINE L.M., 1990. Techniques for the development of transgenic fish : a review UCLA Symposium on Transgenic Models in Medicine and Agriculture. pp. 89-99. Edité par R. Church, Wiley-Liss Inc.

CLARK A J, BESSOS H, BISHOP IO BROWN P., HARRIS S., LATHE R., MC CLENAGHAN M., PROWSE C., SIMONS J.P., WHITELAW C.B.A, WILMUT I., 1989. Expression of human anti-hemophilic factor IX in the milk of transgenic sheep. Biotechnology, 7, 487-492.

CROZET N., 1990. Manipulation of oocytes and in vitro fertilization. J. Reprod. Fert. (sous presse).

GROSCLAUDE F., 1988. Le polymorphisme génétique des principales lacto-protéines bovines. INRA. Prod. Anim., 1, 5-17.

HOUDEBINE L.M., 1990. Les manipulations génétiques : comment améliorer la croissance. INRA. Prod. Anim., 3, 207-214.

JEFFREY A.J., WILSON V., THEIN S.L., 1985. Individual specific fingerprints of human DNA. Nature, 316, 76-79.

JENNINGS P.A., MOLLOY P.L., 1987. Inhibition of SV40 replicase function by engineered antisense RNA transcribed by RNA polymerase III. Embo J. 6, 3043-3046.

LEONARD M., KIRSZENBAUM M., COTINOT C., CHESNE P., HEYMAN Y., STINNAKRE M.G., BISHOP C., DELOUIS C., VAIMAN M., PELLOUS M., 1987. Sexing bovine embryos using $Y$ chromosome specific DNA probe. Theriogenology, 27, 248 .
MANSOUR S.L., THOMAS K.R., CAPECCHI M.R., 1988. Discription of proto- oncogenic int-2 in mouse embryoderived stem cells ; a general strategy for targeting mutations to non-selectable genes. Nature, 336, 348-352.

MARX J.L., 1988. Cloning sheep and cattle embryos. Science, 239, 463-464

MASSEY J.M., 1990. Animal production in the year 2000 A.D. J. Reprod. Fert. Suppl. 41, 199-208.

MASSOUD M., BISCHOFF R., DALEMANS W., POINTU H., ATTAL J., SCHULTZ H., CLESSE D., STINNAKRE M.G., PAVIRANI A., HOUDEBINE L.M., 1990. Expression of active recombinant human $\alpha 1$ antitrypsin in transgenic rabbits. J. Biotech. (sous presse).

Mc GRAN RA. JACOBSON R.J., AKAMATSU M., 1988. A male-specific repeated DNA sequence in the domestic pig. Nucleic Acids Res. 16, 10389.

PETITTE I.N., CLARK M.E., LIU G., GIBBINS A.M., ETCHES R.J., 1990. Production of somatic and germ line chimeras in the chicken by transfer of early blastodermal cells. Development, 108, 185-189.

PRATHER R.S., SIMS M.M., FIRST N.L., 1989. Nuclear transplantation in early pig embryos. Biol. Reprod. 41, 414-418

PURSEL V.G., PINKERT E.A., MILLER K.F, BOLT D.J., CAMPBELL R.G., PALMITER R.D., BRINSTER R.L., HAMMER R.E., 1989. Genetic engineering of livestock. Science, 244, 1281-1288.

REES W.D., FLINT H.J., FULLER M.F, 1990. A molecular biological approach to reducing dietary amino acid needs. Biotechnology, 8, 629-633.

ROBERTSON E., BRADLEY A., KUEHN M., EVANS $M$ 1986. Germ-line transmission of genes introduced into cultured pluripotential cells by retroviral vector. Nature, $323,445-448$

ROGERS G.F.,1990. Improvement of wool production through genetic engineering Tibtech. 8, 6-11.

SANG H., PERRY M.M., 1989. Episomal replication of cloned DNA injected into the fertilised ovum of the hen gallus domesticus. Mol. Reprod. Develop. 1, 98-106.

SARVER N., CANTIN E.M., CHANG P.S., ZAIA I.A. LADNE P.A., STEPHENS D.A., ROSSI J.J., 1990. Ribozymes as potential anti-HIV 1 therapeutic agents Sciences 247, 1222-1225.

\section{Summary}

\section{Animal biotechnologies.}

Recent progress in biology, namely those related to nucleic acids and proteins function offer new very promising strategies for domestic animals breeding. Nucleic acid probes allow sex determination in early bovine embryo. More generally, the numerous probes which are progressively becoming available and genome maping will give a much better knowledge of animals. New strategies for selection which will be more subtle and faster will appear. The bulky preparation of recombinant peptides (antigens from pathogenic agents, hormones, cytokins, monoclonal antibodies...) suggests that new treatments of animals will be used routinely. New techniques related to mammals reproduction: in vitro oocyte maturation, in vitro fertilization, in vitro early embryo development, duplication of embryos through cleavage and cloning) will be progressively added to the already existing techniques (artificial insemination, embryo transfer). They will contribute to shorten the reproductive cycle and selection programmes. Genetic engineering in the best situation will allow to administer a protein to animals through gene transfer to somatic cells (gene therapy) or to early embryos (transgenesis). Gene therapy may give the possibility to be no more obliged to inject a peptide repeatedly whereas transgenesis leads to the generation of new animal breeds exhibiting new genetic properties provided by the transgenes. These methods which are the most potent and the most promising will not become a reality for animal breeding before the end of this century.

HOUDEBINE L.M., 1991. Les biotechnologies animales. INRA, Prod. Anim., 4 (1), 81 - 88. 